\title{
Nonlinear optical tuning of photonic crystal microcavities by near-field probe
}

\author{
Silvia Vignolini, ${ }^{1, a)}$ Francesca Intonti, ${ }^{1,2}$ Laurent Balet, ${ }^{3}$ Margherita Zani, ${ }^{1}$ \\ Francesco Riboli, ${ }_{4}^{1}$ Anna Vinattieri, ${ }^{1}$ Diederik S. Wiersma, ${ }^{1}$ Marcello Colocci, ${ }^{2}$ Lianhe $\mathrm{Li}^{3}{ }^{3}$ \\ Marco Francardi, ${ }^{4}$ Annamaria Gerardino, ${ }^{4}$ Andrea Fiore, ${ }^{5}$ and Massimo Gurioli ${ }^{1}$ \\ ${ }^{1}$ LENS and Department of Physics, University of Florence, Via Nello Carrara 1, \\ 50019 Sesto Fiorentino, Italy \\ ${ }^{2}$ CNISM, Unità di Ricerca di Firenze, Via Sansone 1, 50019 Sesto Fiorentino, Italy \\ ${ }^{3}$ Ecole Polytechnique Fédérale de Lausanne, Institute of Photonics and Quantum Electronics, Station 3, \\ CH-1015 Lausanne, Switzerland \\ ${ }^{4}$ Institute of Photonics and Nanotechnology, CNR, via del Cineto Romano 42, 00156 Roma, Italy \\ ${ }^{5}$ COBRA Research Institute, Eindhoven University of Technology, 5600 MB Eindhoven, The Netherlands
}

(Received 10 May 2008; accepted 30 June 2008; published online 17 July 2008)

\begin{abstract}
We report on a nonlinear way to control and tune the dielectric environment of photonic crystal microcavities exploiting the local heating induced by near-field laser excitation at different excitation powers. The temperature gradient due to the optical absorption results in an index of refraction gradient which modifies the dielectric surroundings of the cavity and shifts the optical modes. Reversible tuning can be obtained either by changing the excitation power density or by exciting in different points of the photonic crystal microcavity. (C) 2008 American Institute of Physics. [DOI: 10.1063/1.2960259]
\end{abstract}

Optical microcavities, in particular photonic crystal microcavities (PC-MCs), are of profound interest in several fields of nanophotonics. ${ }^{1-3} \mathrm{~A}$ crucial issue in the progress of the field is the possibility to control and tune the optical modes. Different approaches have been, so far, used to tune the PC-MCs resonance. ${ }^{4-7}$ Successive oxidation and wet etching cycles have been used for enlarging the radius of holes in the PC and decreasing the membrane thickness; this method allowed a large mode shift up $37 \mathrm{~nm}$, but in a irreversible way. ${ }^{4}$ Gas condensation onto a PC slab MC maintained at low temperature has been used to modify the dielectric mismatch of the PC-MCs and to shift the cavity mode wavelength by as much as $5 \mathrm{~nm}$. The method works, however, only at low temperature. ${ }^{5}$ Local changes of the refractive index of the air holes via infiltration of liquids at the single hole level ${ }^{8}$ have been already demonstrated, but only on pore sizes slightly below one micron. Recently, scanning microscope tip induced shifts of the PCs cavities modes have been studied as well. ${ }^{9-12}$ In this case, the dielectric tip acts as an external nano-object that locally modifies the dielectric environment and tunes the optical mode in a reversible and dynamical way.

In this paper we report on a nonlinear method to control and tune the dielectric environment of PC-MCs by exploiting the local heating induced by near-field laser excitation at different excitation powers. The temperature gradient due to the optical absorption results in an index of refraction gradient which modifies the dielectric surroundings of the cavity and shifts the optical modes. Reversible tuning can be obtained either by changing the excitation power density or by exciting in different points of the MC. We therefore demonstrate a nonlinear enhancement of the reversible tip induced tuning of the optical modes.

The investigated PC-MC incorporates QDs acting as local light sources and is fabricated on a GaAs suspended membrane. Three layers of high-density InAs QDs emitting

\footnotetext{
${ }^{a)}$ Electronic mail: vignolini@lens.unifi.it.
}

at $1300 \mathrm{~nm}$ are grown by molecular beam epitaxy at the center of the 320-nm membrane. The details on the fabrication can be found in Ref. 13. The structure under consideration consists of a two-dimensional triangular lattice of air holes with lattice parameter $a=301 \mathrm{~nm}$ and filling fraction $f=35 \%$, where the cavity is formed by four missing holes organized in a diamond-like geometry. A scanning electron microscope image is reported in Fig. 1(a). A commercial scanning near-field optical microscope (SNOM) is used in an illumination/collection geometry with a combined spatial and spectral resolution of 250 and $0.1 \mathrm{~nm}$, respectively. In this geometry, the sample is excited with light from a diode laser $(780 \mathrm{~nm})$ coupled into a chemically etched, uncoated near-field fibre probe, ${ }^{14}$ that is raster scanned at a constant height on the sample surface. PL spectra from the sample were collected at each tip position through the same probe. Numerical simulations are performed with the finitedifference time-domain (FDTD) method, using a freely available software package (MEEP). ${ }^{15}$

Figure 1(b) shows a typical PL spectrum of the structure under investigation characterized by two main peaks, M1 and M2, centered around 1266.8 and $1241.3 \mathrm{~nm}$, respec-
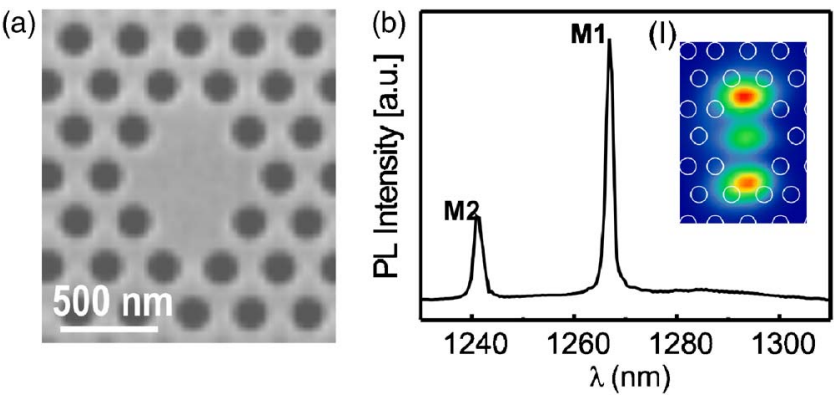

FIG. 1. (Color online) (a) Scanning electron microscope image of the investigated sample. (b) Typical PL spectrum collected by the near-field probe. The inset (I) shows the spatial intensity distribution associated to the mode M1; the white circles superimposed on the image denote the topographic positions of the pores. 

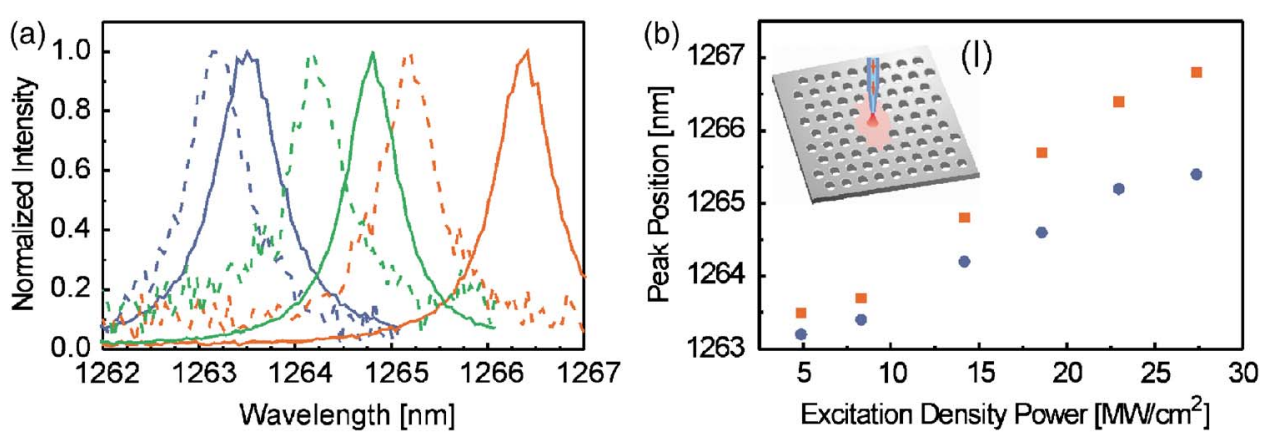

FIG. 2. (Color online) (a) Near-field spectra obtained for different power excitation densities $\left(5,14\right.$, and $23 \mathrm{MW} / \mathrm{cm}^{2}$ in blue, green, and red, respectively) of the mode M1. The spectra reported with a straight line are collected at the vertical edge of the cavity region, while the dotted ones are collected outside the cavity. (b) The dots in the graph represent the peak positions for the spectra presented in (a) as the excitation power is increased. In particular the red squares indicate the peak position of the spectra collected at the vertical edge of the cavity region, while the blue circles indicate the peak position of the spectra collected outside the cavity. The inset (I) provides a schematic representation of the heating mechanism.

tively. In particular, the resonance at longer wavelength is characterized by a FWHM of $0.52 \mathrm{~nm}$, corresponding to a quality factor larger than 2400. The inset (I) of Fig. 1 shows the $1.35 \times 2 \mu \mathrm{m}^{2}$ image of the integrated PL intensity in the wavelength range between 1265 and $1267 \mathrm{~nm}$ (i.e., integrated over the M1 optical mode). This intensity distribution qualitatively reproduces the spatial distribution of the electric field associated to the resonance M1 $1{ }^{16}$ The maximum of the local density of states (LDOS) associated to the mode is in correspondence of the maximum of the PL signal. In the following only the results for the mode M1 will be discussed since we obtained analogous information for the mode M2.

Figure 2(a) reports the spectra of the M1 mode detected at one of the vertical edges of the cavity (full line) compared with the spectra obtained with the tip shifted $0.5 \mu \mathrm{m}$ away from the same position (dashed line) for different excitation density powers (different color lines). The first position corresponds to a maximum of the LDOS for the electric field in the PC-MC associated to the mode M1, while the second position corresponds to the spatial tail of the LDOS associated to the same peak. For sake of simplicity and concision, in the following we will use the denomination of spatially resonant excitation for the case of pumping on the position corresponding to a maximum of LDOS and the denomination of spatially nonresonant excitation for the case of pumping on a point with a low LDOS. The spectrum recorded at the apex of the PC-MC (i.e., in the spatially resonant case) shows a clear redshift with respect to the spectrum recorded outside the PC defect (i.e., in the spatially nonresonant case). At low excitation power, this agrees with the tip induced mode shift associated to the dielectric environment modification of the PC-MCs due to the presence of the near-field tip, as recently observed. ${ }^{11,12}$ In addition, we find that the mode energy and the value of the shift depend on the excitation power, as shown also in Fig. 2(b), where a summary for six different excitation power densities is reported. Fixing the position of the tip with respect to the sample and increasing the excitation power, we observe a monotonic shift of the PC-MC mode. Moreover a larger nonlinear effect is observed for the spatially resonant case. In principle there are two main mechanisms for optical nonlinearities in PC-MCs under laser excitation, which can be easily identified by the sign of the mode shift. The first is related to the index of refraction modification due to free carrier generation. This has been shown to produce ultrafast nonlinear optical tuning of PCMCs under ps laser excitation and it gives a blue shift of the optical mode. ${ }^{17}$ The second nonlinear effect is related to the thermal tuning due to dissipation of the absorbed excitation power and it gives a shift to longer wavelengths of the PC-MC optical mode. In our case, the mode shifts on the red side and, therefore, we attribute the power dependent shift to thermal heating as expected for cw excitation.

Our understanding of the physics behind the observed data is as follows. The laser pumping with a nanometric SNOM tip induces an inhomogeneous heating of the cavity, as schematically illustrated in the inset (I) of Fig. 2(b). Since we are using uncoated SNOM tips, we can completely neglect the effects due to the heating of the probe itself, observed in the case of a metal coated tip ${ }^{18,19}$ where the tip-tosample heat transfer is, however, small. ${ }^{20,21}$ In our case, the heating mechanism is due to the optical absorption in the sample and it creates a change of the index of refraction of the sample and therefore a modification of the dielectric environment of the PC-MCs. The thermal gradient is enhanced due to the reduced heat diffusion out of the PC membrane. In order to map the thermal gradient created in this configuration, we report in Fig. 3(a) the spatial decay of the spectral shift of the cavity mode as the excitation density power is increased. For low excitation power (blue dots), the decay constant associated to the mode spectral shift is comparable with the one associated to the PL intensity (black squares). By assuming an exponential decay of the mode we obtain, in this case, a decay constant of roughly $150 \pm 50 \mathrm{~nm}$. In this case, the heating effect could be neglected and the map of the spectral shift reproduces the map of the electric field intensity associated to the mode. ${ }^{12}$ For higher excitation powers, the profile of the mode shift broadens due to the thermal gradient and we observe a reduction of the spectral shift of $1.3 \mathrm{~nm}$ by moving the SNOM tip by $500 \mathrm{~nm}$. This result indicates that with this method we are able to obtain information about the spatial distribution of the temperature averaged over the cavity mode volume.

In order to interpret in a quantitative manner the experimental results, we can consider, at least in the case of spatially non resonant excitation and given to the small volume of the PC-MC mode, an effective average temperature and neglect the thermal gradient. The thermal tuning of the optical mode has been then quantified by performing low excitation measurements at different temperatures by heating the PC-MC with a resistor. By linearly fitting the dependence of the mode spectral position on the temperature, we obtain a thermal tuning of $0.12 \mathrm{~nm} / \mathrm{K}$. This means that the $2.1 \mathrm{~nm}$ license or copyright; see http://apl.aip.org/about/rights_and_permissions 

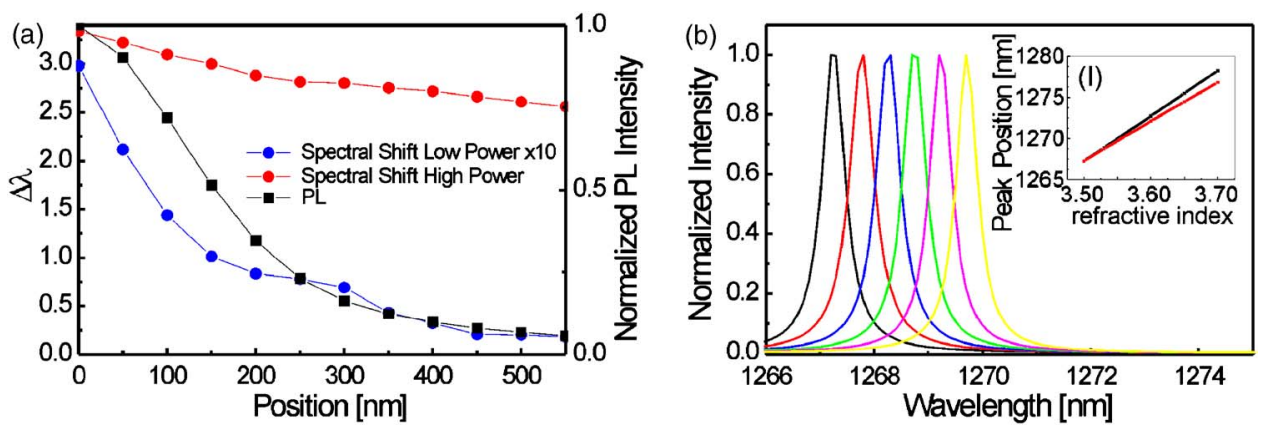

FIG. 3. (Color online) (a) Spectral shift spatial decay of the cavity mode for a excitation density power of $5 \mathrm{MW} / \mathrm{cm}^{2}$ (blue dots, scaled by a factor 10 ) and $27 \mathrm{MW} / \mathrm{cm}^{2}$ (red dots). The decay rate for the case of low excitation density power is comparable with the PL spatial decay associated to the same mode (black squares, right axis). (b) Calculated FDTD spectra obtained inserting a dielectric cylinder (diameter of $300 \mathrm{~nm}$ ) in the center of the cavity for different values of refractive index from 3.50 to 3.55 at step of 0.01 . As the refractive index is increased the mode is redshifted. The inset reports the spectral position of the mode as a function of the refractive index of the cylinder placed in the center of the cavity (red line) and on the vertical apex of the PC-MC.

maximum shift experimentally observed under nonresonant excitation would correspond to a global heating of the PCMCs of the order of $18 \mathrm{~K}$. From FDTD simulations, a redshift of $2.1 \mathrm{~nm}$ can be retrieved by a global increase of the index of refraction of $\Delta n=0.006$ which gives a linear dependence of the index of refraction $\Delta n / \Delta T=3.3 \times 10^{-4} \mathrm{~K}^{-1}$ to be compared with the value of $\Delta n / \Delta T=2.710^{-4} \times \mathrm{K}^{-1}$ reported in the literature for GaAs in the case of vertical cavity surface type optical resonator at wavelength around $1 \mu \mathrm{m} .{ }^{22}$

In the case of spatially resonant excitation, the nonhomogeneous heating of the PC-MC cannot be neglected. In order to evaluate it, we consider the limit case of an additional hot spot in correspondence of the tip position that spatially decays over a distance comparable with the SNOM resolution $(300 \mathrm{~nm})$. When the hot spot is created in correspondence of a maximum of LDOS of the PC-MCs the photogenerated gradient of index of refraction strongly modifies the shape of the dielectric defect, resulting in a slightly different PC-MC. In the FDTD calculation, we simulate this effect by using an additional cylinder of larger index of refraction $(\Delta n)$ placed in different positions of the PC-MC. Within this crude model, it is possible to obtain a different spectral shift of the mode for different combinations of the dielectric cylinder diameter $\mathbf{d}$ and $\Delta n$. Figure $3(\mathrm{~b})$ reports the simulated spectra obtained by placing a dielectric cylinder with $\mathbf{d}=300 \mathrm{~nm}$ in the center of the cavity and increasing at steps of 0.01 the values of its refractive index from 3.5 (that is the same value of refractive index of the cavity), to 3.55 . The inset (I) of Fig. 3(b) shows the behavior of the peak position as the refractive index is increased in the case where the dielectric cylinder is placed in the center or at the vertical edge of the cavity from a value of refractive index of 3.55 to 3.75. The peak position moves to larger wavelengths with the increase of the refractive index and the slope depends on the overlap between the spatial electric field distribution associated to mode and the position of the cylinder. The theoretical results reproduce the measured spatially resonant shift if we assume an increase of $\Delta n=0.03$ in the refractive index of the dielectric cylinder, corresponding to a local heating of roughly $100 \mathrm{~K}$. Note that the optical shift of the mode is roughly proportional to the hot spot diameter (at least for $\mathbf{d}$ smaller that the mode volume); for $\mathbf{d}=400 \mathrm{~nm}$ the local heating decreases to $75 \mathrm{~K}$.

Financial support is acknowledged from the MIURPRIN project (2005025173), the Swiss National Science
Foundation (Professeur boursier program), the Swiss State Secretariate for Education and Research through COST-P11 Project C05-70, EU-FP6 IP "QAP” (Contract No. 15848), and the Italian MIUR-FIRB program.

${ }^{1}$ W.-H. Chang, W.-Y. Chen, H.-S. Chang, T.-P. Hsieh, J.-I. Chyi, and T.-M. Hsu, Phys. Rev. Lett. 96, 127404 (2006).

${ }^{2}$ H.-G. Park, S.-H. Kim, S.-H. Kwon, Y.-G. Ju, J.-K. Yang, J.-H. Baek, S.-B. Kim, and Y.-H. Lee, Science 305, 1444 (2004).

${ }^{3}$ K. Hennessy, A. Badolato, M. Winger, D. Gerace, M. Atatüre, S. Gulde, S. Fält, E. L. Hu, and A. Imamoğlu, Nature (London) 445, 896 (2007).

${ }^{4}$ D. Dalacu, S. Frédérick, P. J. Poole, G. C. Aers, and R. L. Williams, Appl. Phys. Lett. 87, 151107 (2005).

${ }^{5}$ S. Mosor, J. Hendrickson, B. C. Richards, J. Sweet, G. Khitrova, H. M. Gibbs, T. Yoshie, and A. Scherer, Appl. Phys. Lett. 87, 141105 (2005).

${ }^{6}$ S. Strauf, M. T. Rakher, I. Carmeli, K. Hennessy, C. Meier, A. Badolato, M. J. A. De Dood, P. M. Petroff, E. L. Hu, E. G. Gwinn, and D. Bouwmeester, Appl. Phys. Lett. 88, 043116 (2006).

${ }^{7}$ K. Hennessy, C. Högerle, E. Hu, A. Badolato, and A. Imamoglu, Appl. Phys. Lett. 89, 041118 (2006).

${ }^{8}$ F. Intonti, S. Vignolini, V. Türck, M. Colocci, P. Bettotti, L. Pavesi, S. L. Schweizer, R. Wehrspohn, and D. Wiersma, Appl. Phys. Lett. 89, 211117 (2006).

${ }^{9}$ S. Mujumdar, A. F. Koenderink, T. Sunner, B. C. Buchler, M. Kamp, A. Forchel, and V. Sandoghdar, Opt. Express 15, 17214 (2007).

${ }^{10}$ I. Märki, M. Salt, and H. P. Herzig, Opt. Express 14, 2969 (2006).

${ }^{11}$ W. C. L. Hopman, A. J. F. Hollink, R. M. de Ridder, K. O. van der Werf, V. Subramaniam, and W. Bogaerts, Opt. Express 14, 8745 (2006).

${ }^{12}$ F. Intonti, S. Vignolini, F. Riboli, A. Vinattieri, D. S. Wiersma, M. Colocci, L. Balet, C. Monat, C. Zinoni, L. H. Li, R. Houdré, M. Francardi, A. Gerardino, A. Fiore, and M. Gurioli, Phys. Rev. B (unpublished).

${ }^{13}$ M. Francardi, L. Balet, A. Gerardino, C. Monat, C. Zinoni, L. H. Li, B. Alloing, N. Le Thomas, R. Houdré, and A. Fiore, Phys. Status Solidi C 3, 3693 (2006).

${ }^{14}$ R. Stoeckle, C. Fokas, V. Deckert, R. Zenobi, B. Sick, B. Hecht, and U. P. Wild, Appl. Phys. Lett. 75, 160 (1999).

${ }^{15}$ A. Farjadpour, D. Roundy, A. O. Rodriguez, M. Ibanescu, P. Bermel, J. D. Joannopoulos, S. G. Johnson, and G. Burr, Opt. Lett. 31, 2972 (2006).

${ }^{16}$ F. Intonti, S. Vignolini, F. Riboli, A. Vinattieri, D. S. Wiersma, M. Colocci, M. Gurioli, L. Balet, C. Monat, L. H. Li, N. Le Thomas, R. Houdre, A. Fiore, M. Francardi, A. Gerardino, F. Roemer, and B. Witzigmann, Physica E (Amsterdam) 40, 1965 (2008).

${ }^{17}$ I. Fushman, E. Waks, D. Englund, N. Stoltz, P. Petroff, and J. Vučković, Appl. Phys. Lett. 90, 091118 (2007).

${ }^{18}$ A. H. La Rosa, B. I. Yakobson, and H. D. Hallen, Appl. Phys. Lett. 67, 2597 (1995).

${ }^{19}$ M. Stähelin, M. A. Bopp, G. Tarrach, A. J. Meixner, and I. ZschokkeGränacher, Appl. Phys. Lett. 68, 2603 (1996).

${ }^{20}$ D. Kazantsev, G. Guttroff, M. Bayer, and A. Forchel, Appl. Phys. Lett. 72, 689 (1998).

${ }^{21}$ P. G. Gucciardi, S. Patanè, A. Ambrosio, M. Allergini, A. D. Dounes, G. Latini, O. Fenwich, and F. Cacialli, Appl. Phys. Lett. 86, 203109 (2005).

${ }^{22}$ J. Talghader and J. S. Smith, Appl. Phys. Lett. 66, 335 (1995). 